\title{
Modified frailty index predicts complications and death after non-bariatric gastrectomies
}

\author{
Konstantinos A. Zorbas ${ }^{1}$, Vic Velanovich ${ }^{2}$, Nestor F. Esnaola ${ }^{3}$, Andreas Karachristos ${ }^{4}$ \\ ${ }^{1}$ Department of Surgery, Bronx Care Health System, New York, NY, USA; ${ }^{2}$ Division of General Surgery, Department of Surgery, University of South \\ Florida, Tampa, FL, USA; ${ }^{3}$ Division of Surgical Oncology, Department of Surgery, Houston Methodist Hospital, Houston, Texas, USA; ${ }^{4}$ Division of \\ Surgical Oncology, Department of Surgery, University of South Florida, Tampa, FL, USA \\ Contributions: (I) Conception and design: KA Zorbas, A Karachristos; (II) Administrative support: KA Zorbas, A Karachristos; (III) Provision of \\ study materials or patients: KA Zorbas, A Karachristos; (IV) Collection and assembly of data: KA Zorbas, A Karachristos; (V) Data analysis and \\ interpretation: All authors; (VI) Manuscript writing: All authors; (VII) Final approval of manuscript: All authors. \\ Correspondence to: Andreas Karachristos. Division of Surgical Oncology, Department of Surgery, University of South Florida, Tampa, FL, USA. \\ Email: andreask@usf.edu.
}

\begin{abstract}
Background: The modified frailty index $(\mathrm{mFI})$ has been shown to predict mortality and morbidity after major operations. The aim of the present study was to assess the $\mathrm{mFI}$ as a preoperative predictor of shortterm postoperative complications and 30-day mortality in patients undergoing gastrectomy for non-bariatric diseases.

Methods: The American College of Surgeons National Surgical Quality Improvement Program (ACS NSQIP) database was queried for patients who underwent total or partial gastrectomy from 2005 to 2011. A $\mathrm{mFI}$ was calculated based on 11 variables as previously described. The population divided into the following four categories based on the mFI score: the non-frail (mFI 0), the low frail (mFI 1), the intermediate frail (mFI 2$)$ and frail $(\mathrm{mFI} \geq 3)$. Thirty-day mortality and postoperative complications were evaluated.

Results: Overall, 5,711 patients underwent a gastrectomy for non-bariatric diseases. Higher mFI score was associated with higher rates of mortality (from $1.2 \%$ in the non-frail group to $10.7 \%$ in frail group, $\mathrm{P}<0.001$ ), overall morbidity $(26.7 \%$ vs. $51.1 \%, \mathrm{P}<0.001)$, postoperative Clavien IV complication $(6 \%$ vs. $24.6 \%$, $\mathrm{P}<0.001)$, serious complications (19.3\% vs. $42.6 \%, \mathrm{P}<0.001)$, sepsis-related complications (8.4\% vs. $16.4 \%$, $\mathrm{P}<0.001)$, cardiopulmonary complications ( $5 \%$ vs. $20.7 \%, \mathrm{P}<0.001)$ and failure to rescue $(5.7 \%$ vs. $21.8 \%$, $\mathrm{P}<0.001)$.

Conclusions: Higher mFI score in patients undergoing non-bariatric gastrectomy, is associated with a stepwise greater risk of postoperative morbidity and mortality. MFI Score can be easily calculated preoperatively, from the patient's history, and it can be used as an exceptionally useful criterion for treatment planning.
\end{abstract}

Keywords: Gastrectomy; outcomes; modified frailty index (mFI); failure to rescue; NSQIP

Received: 21 September, 2019; Accepted: 18 January, 2020; Published: 05 January 2021.

doi: $10.21037 / \operatorname{tgh} .2020 .01 .07$

View this article at: http://dx.doi.org/10.21037/tgh.2020.01.07

\section{Introduction}

Total or subtotal gastrectomy is the only curative treatment for certain malignancies of the stomach. It is still considered to be a major surgical procedure with substantial perioperative morbidity and mortality. Patients undergoing surgery for gastric malignancy are usually older with many comorbidities and hence with higher odds of postoperative complications (1). The rate of postoperative serious complications after gastrectomy is around $24 \%$, and the overall mortality has been estimated between $4-5 \%$ $(2,3)$. However, frail patients might have higher rates of 
postoperative complications compared with the non-frail patients of the same age. The outcomes of gastrectomy in frail patients have not been adequately studied, and outcome predictors for gastrectomy have not been published, other than isolated risk factors (2).

Several authors have reported comorbidities as relative contraindications for gastrectomy without calculating the cumulative effect of several coexisting comorbidities in the postoperative complication rate of any patient. Conventional risk assessment scores such as the American Society of Anesthesiologist (ASA) score or the Postoperative Score to Predict Postoperative Mortality (POSPOM) are of limited clinical use; either because they include a small number of preoperative factors or because they are too complex (4). Obviously, there is an imperative need for studying multiple preoperative clinical risk factors and developing a simple, objective and reliable risk assessment score which would estimate the patient's cumulative health deficit. Recent studies have explored the role of frailty in estimating the postoperative risk after different types of operations. A practical way of frailty measurement is the modified frailty index (mFI), which was first described by Velanovich et al. (5). Although several studies exist on $\mathrm{mFI}$ score as a surgical risk calculator, there are no studies on $\mathrm{mFI}$ score for patients undergoing gastrectomy for nonbariatric diseases.

In this study, we evaluated the utility of the mFI score as a surgical risk estimator for postoperative complication by using the NSQIP database. We also evaluated the association of each individual frailty components with postoperative serious complications and mortality. We hypothesized that mFI score would be able to identify patients at high risk for short-term (30 days) postoperative complications, and it would be a useful tool for preoperative surgical risk stratification. Our primary hypothesis was that higher frailty levels would be associated with higher rates of post-gastrectomy complications and mortality.

\section{Methods}

This is a retrospective cohort analysis of prospectively collected data from the ACS-NSQIP database. The ACS-NSQIP is a national quality improvement program with participation of more than 600 hospitals in 49 of the 50 states. Data is collected on over 150 variables, including preoperative risk factors, intraoperative variables, and postoperative mortality and morbidity (6).

The NSQIP participant use files were queried from 2005-
2011 for patients who underwent partial or total gastrectomy, either open or laparoscopic for either malignant or benign disease of the stomach. The patients were identified based on primary Current Procedural Terminology (CPT) codes: 43620 (Gastrectomy, total; with esophagoenterostomy), 43621 (Gastrectomy, total; with Roux-en-Y reconstruction), 43622 (Gastrectomy, total; with formation of intestinal pouch, any type), 43631(Gastrectomy, partial, distal; with gastroduodenostomy), 43632 (Gastrectomy, partial, distal; with gastrojejunostomy), 43633 (Gastrectomy, partial, distal; with Roux-en-Y reconstruction) and 43634 (Gastrectomy, partial, distal; with formation of intestinal pouch). Our exclusion criteria were cases with no available data on $\mathrm{mFI}$ score variables, cases with prior operation within 30 days, cases with open wound or wound infection preoperatively, and cases with concurrent other major operations like liver resections, pancreatectomies, splenectomies, colectomies or small bowel resections.

All outcomes were monitored in the first 30 postoperative days after gastrectomy. MFI score was calculated based on methodology recently described by Velanovich et al. (5). Every patient was allotted one point for each of the following preoperative comorbidity if abnormal: (I) patient functional status (dependent or not dependent); (II) diabetes mellitus (treated with insulin or oral medications); (III) hypertension (requiring treatment); (IV) congestive heart failure (CHF); (V) myocardial infarction (MI); (VI) either prior cardiac surgery and/or percutaneous coronary angioplasty and/or history of angina; (VII) chronic obstructive pulmonary disease (COPD) and/or history of pneumonia; (VIII) rest pain/gangrene secondary to peripheral vascular disease (PVD) and/or PVD with treatment (angioplasty or revascularization or amputation); (IX) impaired sensorium within 48 hours prior to the surgical procedure (not in the context of current neurologic disease like dementia); (X) history of transient ischemic attack (TIA) and/or cerebrovascular accident (CVA) without neurologic deficit and (XI) CVA with neurologic deficit. The total $\mathrm{mFI}$ was calculated for every patient. The possible $\mathrm{mFI}$ score range from zero to eleven, but in our study population the $\mathrm{mFI}$ score range from zero to seven (Table 1). For ease of interpretation and easier future clinical use, the total population was divided into four groups to determine the relationship of frailty to mortality, morbidity and failure to rescue. This was a similar method described by Augustin et al. (7). The four groups of $\mathrm{mFI}$ are the following: no frailty group (with $\mathrm{mFI}$ score zero), low frailty group (with $\mathrm{mFI}$ score 1), intermediate frailty group (with $\mathrm{mFI}$ score 2 ) 
Table 1 Mortality, Clavien IV complications and failure to rescue distribution of patients undergoing gastrectomy based on modified frailty index score

\begin{tabular}{|c|c|c|c|c|}
\hline Modified frailty index & Total, n (\%) & Mortality, n (\%) & Clavien IV complications ${ }^{1}, \mathrm{n}(\%)$ & Failure to rescue ${ }^{2}, \mathrm{n}(\%)$ \\
\hline Overall & 5,711 & $221(3.9)$ & $635(11.1)$ & $189(13.1)$ \\
\hline 0 & $2,101(36.8)$ & $25(1.2)$ & $126(6.0)$ & $23(5.7)$ \\
\hline 1 & $1,922(33.7)$ & $67(3.5)$ & $191(9.9)$ & $53(11.5)$ \\
\hline 2 & $1,073(18.8)$ & $63(5.9)$ & $167(15.6)$ & $56(17.6)$ \\
\hline 3 & $412(7.2)$ & $37(9.0)$ & $96(23.3)$ & $30(17.9)$ \\
\hline 4 & $143(2.5)$ & $19(13.3)$ & $37(25.9)$ & $18(28.6)$ \\
\hline 5 & $45(0.8)$ & $5(11.1)$ & $11(24.4)$ & $4(17.4)$ \\
\hline 6 & $11(0.2)$ & $3(27.3)$ & $5(45.5)$ & $3(50.0)$ \\
\hline 7 & $4(0.1)$ & $2(50.0)$ & $2(50.0)$ & $2(100.0)$ \\
\hline
\end{tabular}

${ }^{1}$, Clavien IV complications is any one of the following complications in the NSQIP database: cardiac arrest requiring CPR; myocardial infarction; pulmonary embolism; postoperative acute renal failure requiring dialysis; septic shock; failure to wean from ventilator $>48$ hours and postoperative need for unplanned reintubation. ${ }^{2}$, failure to rescue is defined as death after serious complication.

and frail group (with $\mathrm{mFI}$ score $\geq 3$ ) (Table 2).

Preoperative patient demographic, clinical and laboratory characteristics were analyzed in an attempt to investigate for possible confounding factors. The analyzed preoperative variables were age, gender, race, body mass index (BMI), ASA score $\geq 3$, preoperative body weight loss $>10 \%$ in the last six months, preoperative chronic steroid use, postoperative diagnosis (malignancy $v s$. benign disease), preoperative chemotherapy, preoperative radiotherapy, type of gastrectomy, preoperative platelets $\leq 50 \times 10^{3} / \mathrm{mm}^{3}$ and preoperative hematocrit $<30 \%$ (Table 2). BMI and age were analyzed as continuous variables, while all the other variables were analyzed as categorical (Table 2).

Postoperative outcomes assessed were: 30-day mortality, overall morbidity, Clavien IV complications (CLIVC), cardiopulmonary complications (included postoperative pulmonary embolism and/or cardiac arrest and/or MI and/ or failure to wean), sepsis-related complications (included postoperative sepsis and/or septic shock), organ space surgical site infection (SSI), serious postoperative complications and failure to rescue after a serious complication (Table 3). CLIVC are life-threatening complications that lead to single organ or multi-organ dysfunction, requiring intensive care unit (ICU) management (8). In our study we defined CLIVC as any one of the following complications in the NSQIP database: cardiac arrest requiring CPR; MI; pulmonary embolism; postoperative acute renal failure requiring dialysis; septic shock; failure to wean from ventilator $>48$ hours, and postoperative need for unplanned reintubation. Serious complications is defined as (9): pneumonia, unplanned intubation, pulmonary embolism, failure to wean from ventilation, acute renal failure, cerebrovascular accident/ stroke with neurological deficit, MI, cardiac arrest requiring CPR, organ space surgical site infection, deep incisional surgical site infection, wound disruption, sepsis, septic shock, bleeding which needed transfusion and progressive renal insufficiency. Failure to rescue was defined as death after serious complication $(9,10)$. Organ space SSI rates were used as a surrogate for postoperative anastomotic leak in order to evaluate whether the postoperative mortality and complications were associated with the technical failure or with patient's lower physiologic reserves.

Statistical analysis was performed using the program SPSS Statistics Version 24. Data on categorical variables were expressed as frequencies and proportions (\%) and were compared between groups of frailty using the ChiSquare test. Data on continuous variables were summarized with descriptive statistics such as means and standard deviations (SDs), and group comparisons of these variables were performed using the ANOVA test. Univariable logistic regression models were used to examine the associations of frailty and other individual predictor variables with the CLIVC and mortality one at a time. Furthermore, multivariable logistic regression was performed to understand the relationship of frailty with the odds of patients having a CLIVC or mortality, after controlling the statistically significant factors in the univariate analysis. Age and BMI transformed to categorical variables in univariate 
Table 2 Preoperative demographic characteristics and comorbidities

\begin{tabular}{|c|c|c|c|c|c|c|}
\hline Variable & $\begin{array}{c}\text { Total } \\
\text { population, } \\
n=5,711(100 \%)\end{array}$ & $\begin{array}{l}\text { No frailty } \\
m F I=0 \\
n=2,101 \\
(36.8 \%)\end{array}$ & $\begin{array}{l}\text { Low frailty } \\
\text { mFI =1, } \\
n=1,922 \\
(33.7 \%)\end{array}$ & $\begin{array}{c}\text { Intermediate } \\
\text { frailty } \mathrm{mFI}=2, \\
\mathrm{n}=1,073(18.8 \%)\end{array}$ & $\begin{array}{c}\text { Frail } m F I \geq 3 \\
n=615(10.8 \%)\end{array}$ & $\mathrm{P}$ value \\
\hline Age, mean ( \pm SD) (missing: 75/1.3 \%) & $62.3(14.5)$ & $54.6(13.8)$ & $64.7(13.4)$ & $68.3(11.8)$ & $71.3(10.7)$ & $<0.001$ \\
\hline Age $\geq 65(\%)$ & $2,668(46.7)$ & $496(23.7)$ & $1,034(54.5 \%)$ & $678(65.0)$ & $460(76.5)$ & $<0.001$ \\
\hline Gender female, $\mathrm{n}(\%)$ (missing: 7/0.1\%) & $2,929(51.3)$ & $1,159(55.3)$ & $1,026(53.4)$ & $509(47.5)$ & $235(38.2)$ & $<0.001$ \\
\hline Race Caucasian, n (\%) (missing: 516/9\%) & $3,814(66.8)$ & $1,395(66.4)$ & $1,278(66.5)$ & $728(67.8)$ & $413(67.2)$ & 0.85 \\
\hline BMI, mean ( \pm SD) (missing: 121/2.1\%) & $27.54(7.98)$ & $26.3(7.8)$ & $27.83(7.96)$ & $29(8.37)$ & $28.3(7.27)$ & $<0.001$ \\
\hline$A S A \geq 3, n(\%)$ (missing: $8 / 0.1 \%)$ & $3,848(67.5)$ & $1,013(48.3)$ & $1,333(69.5)$ & $912(85.2)$ & $590(95.9)$ & $<0.001$ \\
\hline Weight loss, $\mathrm{n}(\%)$ (missing: 0/0\%) & $794(13.9)$ & $331(15.8)$ & $281(14.6)$ & $116(10.8)$ & $66(10.7)$ & $<0.001$ \\
\hline Steroid use, $\mathrm{n}(\%)$ (missing: 0/0\%) & $163(2.9)$ & $35(1.7)$ & $46(2.4)$ & $42(3.9)$ & $40(6.5)$ & $<0.001$ \\
\hline Patients with malignancy, $\mathrm{n}(\%)$ (missing: 0/0\%) & $3,317(58.1)$ & $1,161(55.3)$ & $1,151(59.9)$ & $645(60.1)$ & $360(58.5)$ & 0.01 \\
\hline Chemotherapy, n (\%) (missing: 0/0\%) & $237(4.1)$ & $103(4.9)$ & $77(4.0)$ & $39(3.6)$ & $18(2.9)$ & 0.106 \\
\hline Radiotherapy, n (\%) (missing: 8/0.1\%) & $104(1.8)$ & $48(2.3)$ & $27(1.4)$ & $20(1.9)$ & $9(1.5)$ & 0.185 \\
\hline Type of operation & & & & & & $<0.001$ \\
\hline Distal/partial gastrectomy & $4,175(73.1)$ & $1,457(69.3)$ & $1,411(73.4)$ & $821(76.5)$ & $486(79)$ & \\
\hline Total gastrectomy & $1,326(23.2)$ & $557(26.5)$ & $444(23.1)$ & $211(19.7)$ & $114(18.5)$ & \\
\hline Gastrectomy with pouch formation & $210(3.7)$ & $87(4.1)$ & $67(3.5)$ & $41(3.8)$ & $15(2.4)$ & \\
\hline Platelets $\leq 50, n(\%)$ (missing: 205/3.6\%) & $30(0.5)$ & $14(0.7)$ & $9(0.5)$ & $3(0.3)$ & $4(0.7)$ & 0.5 \\
\hline Hematocrit <30, n (\%) (missing: 199/3.5 \%) & $850(15.4)$ & $254(12.6)$ & $293(15.8)$ & $162(15.6)$ & $141(23.5)$ & $<0.001$ \\
\hline
\end{tabular}

BMI, body mass index; ASA score, American Society of Anesthesiologists' (ASA) classification of Physical Health Score. Weight loss: patients with a greater than $10 \%$ decrease in body weight in the six-month interval immediately preceding surgery as manifested by serial weights in the chart, as reported by the patient, or as evidenced by change in clothing size or severe cachexia. Patients who have intentionally lost weight as part of a weight reduction program do not qualify. Steroid use: patient who required regular administration of oral or parenteral corticosteroid medications (e.g., Prednisone, Decadron) in the 30 days prior to surgery for a chronic medical condition (e.g., COPD, asthma, rheumatologic disease, rheumatoid arthritis, inflammatory bowel disease). Topical corticosteroids applied to the skin or corticosteroids administered by inhalation or rectally are not included. Patients who only receive short course steroids (duration 10 days or less) in the $\mathbf{3 0}$ days prior to surgery are not included. Chemotherapy: if the patient had any chemotherapy treatment for cancer in the 30 days prior to surgery. Chemotherapy may include, but is not restricted to, oral and parenteral treatment with chemotherapeutic agents for malignancies such as colon, breast, lung, head and neck, and gastrointestinal solid tumors as well as lymphatic and hematopoietic malignancies such as lymphomas, leukemia, and multiple myeloma. Patient is not included if treatment consists solely of hormonal therapy. Radiotherapy: if the patient had any radiotherapy treatment for cancer in the 90 days prior to surgery. Count If the patient had radiation seeds implanted and the implantation was within 90 days prior to the operation.

and multivariable analysis for easier future clinical use. Patients were categorized in those with age 65 or older and patients with age $<65$. Respectively patients were grouped in those with BMI 30 or higher and patients with $\mathrm{BMI}<30$. Wald tests were used to assess the significant contribution of each predictor variables, and unadjusted raw or adjusted odds ratios and their $95 \%$ confidence intervals (CIs) were reported as appropriate for both CLIVC and mortality. Two-tailed $\mathrm{P}$ values of less than 0.05 were deemed statistically significant. Finally, the impact of every individual frailty component was studied, and the odds of CLIVC and mortality calculated for each particular mFI 
Table 330 days mortality, overall morbidity, Clavien IV complications, cardiopulmonary complications, sepsis related complications, serious complications, failure to rescue and organ space surgical site infections (SSI)

\begin{tabular}{|c|c|c|c|c|c|c|}
\hline Variable, n (\%) & Total cohort & No frailty & Low frailty & $\begin{array}{l}\text { Intermediate } \\
\text { frailty }\end{array}$ & Frail & $P$ value \\
\hline $\mathrm{N}(\%)$ & $5,711(100.0)$ & $2,101(36.8)$ & $1,922(33.7)$ & $1,073(18.8)$ & $615(10.8)$ & \\
\hline Mortality & $221(3.9)$ & $25(1.2)$ & $67(3.5)$ & $63(5.9)$ & $66(10.7)$ & $<0.001$ \\
\hline Overall morbidity & $1867(32.7)$ & $562(26.7)$ & $594(30.9)$ & $397(37.0)$ & $314(51.1)$ & $<0.001$ \\
\hline Clavien IV complications & $635(11.1)$ & $126(6.0)$ & $191(9.9)$ & $167(15.6)$ & $151(24.6)$ & $<0.001$ \\
\hline Cardiopulmonary complications & $518(9.1)$ & $106(5.0)$ & $149(7.8)$ & $136(12.7)$ & $127(20.7)$ & $<0.001$ \\
\hline Sepsis related complications & $598(10.5)$ & $176(8.4)$ & $191(9.9)$ & $130(12.1)$ & $101(16.4)$ & $<0.001$ \\
\hline Serious complications & $1,446(25.3)$ & 405 (19.3) & $461(24.0)$ & $318(29.6)$ & $262(42.6)$ & $<0.001$ \\
\hline Failure to rescue & $189(13.1)$ & $23(5.7)$ & $53(11.5)$ & $56(17.6)$ & $57(21.8)$ & $<0.001$ \\
\hline Organ space SSI & $328(5.7)$ & $117(5.6)$ & $98(5.1)$ & $67(6.2)$ & $46(7.5)$ & 0.138 \\
\hline \multicolumn{7}{|l|}{ Patients with malignancy } \\
\hline N (\%) & $3,317(100.0)$ & $1,161(35.0)$ & $1,151(34.7)$ & $645(19.4 \%)$ & $360(10.9)$ & \\
\hline Mortality & $102(3.1)$ & $14(1.2)$ & $34(3.0)$ & $32(5.0)$ & $22(6.1)$ & $<0.001$ \\
\hline Overall morbidity & $1,019(30.7)$ & $313(27.0)$ & $337(29.3)$ & $209(32.4)$ & $160(44.4)$ & $<0.001$ \\
\hline Clavien IV complications & $300(9.0)$ & $69(5.9)$ & $92(8.0)$ & $76(11.8)$ & $63(17.5)$ & $<0.001$ \\
\hline Cardiopulmonary complications & $227(6.8)$ & $56(4.8)$ & $65(5.6)$ & $60(9.3)$ & $46(12.8)$ & $<0.001$ \\
\hline Failure to rescue & $96(14.4)$ & $9(5.0)$ & $23(11.5)$ & $26(16.6)$ & $38(28.8)$ & $<0.001$ \\
\hline Organ space SSI & $190(5.7)$ & $69(5.9)$ & $56(4.9)$ & $39(6.0)$ & $26(7.2)$ & 0.348 \\
\hline \multicolumn{7}{|l|}{ Patients with benign disease } \\
\hline $\mathrm{N}(\%)$ & 2,394 (100.0) & $940(39.3)$ & $771(32.2)$ & $428(17.9)$ & $255(10.7)$ & \\
\hline Mortality & $119(5.0)$ & $11(1.2)$ & $33(4.3)$ & $31(7.2)$ & $44(17.3)$ & $<0.001$ \\
\hline Overall morbidity & $848(35.4)$ & $249(26.5)$ & $257(33.3)$ & $188(43.9)$ & $154(60.4)$ & $<0.001$ \\
\hline Clavien IV complications & $335(14)$ & $57(6.1)$ & $99(12.8)$ & $91(21.3)$ & $88(34.5)$ & $<0.001$ \\
\hline Cardiopulmonary complications & $291(12.2)$ & $50(5.3)$ & $84(10.9)$ & $76(17.8)$ & $81(31.8)$ & $<0.001$ \\
\hline Failure to rescue & $116(4.6)$ & $16(1.6)$ & $40(4.8)$ & $35(8.1)$ & $25(9.9)$ & $<0.001$ \\
\hline Organ space SSI & $138(5.8)$ & $48(5.1)$ & $42(5.4)$ & $28(6.5)$ & $20(7.8)$ & 0.334 \\
\hline
\end{tabular}

component.

\section{Results}

\section{Baseline characteristics of the population}

The final population after applying the exclusion criteria was 5,711 patients (Figure 1). There were 2,101 (36.8\%) patients with no frailty, 1,922 (33.7\%) patient with low frailty (mFI score 1), 1,073 (18.8\%) with intermediate frailty (mFI score 2) and $615(10.8 \%)$ frail patients (mFI score $\geq 3$ ) (Table 2). The mean age of the population was $62.3( \pm 14.5)$ and higher $\mathrm{mFI}$ groups had higher means of age compared to the non-frail group. The overall BMI was $27.5( \pm 7.98)$ with significantly higher BMI patients in the intermediate and frail groups. Of the total population, 2,929 $(51.3 \%)$ patients were female, and 3,814 (66.8) patients were Caucasian. Higher frailty groups include increased rates of 


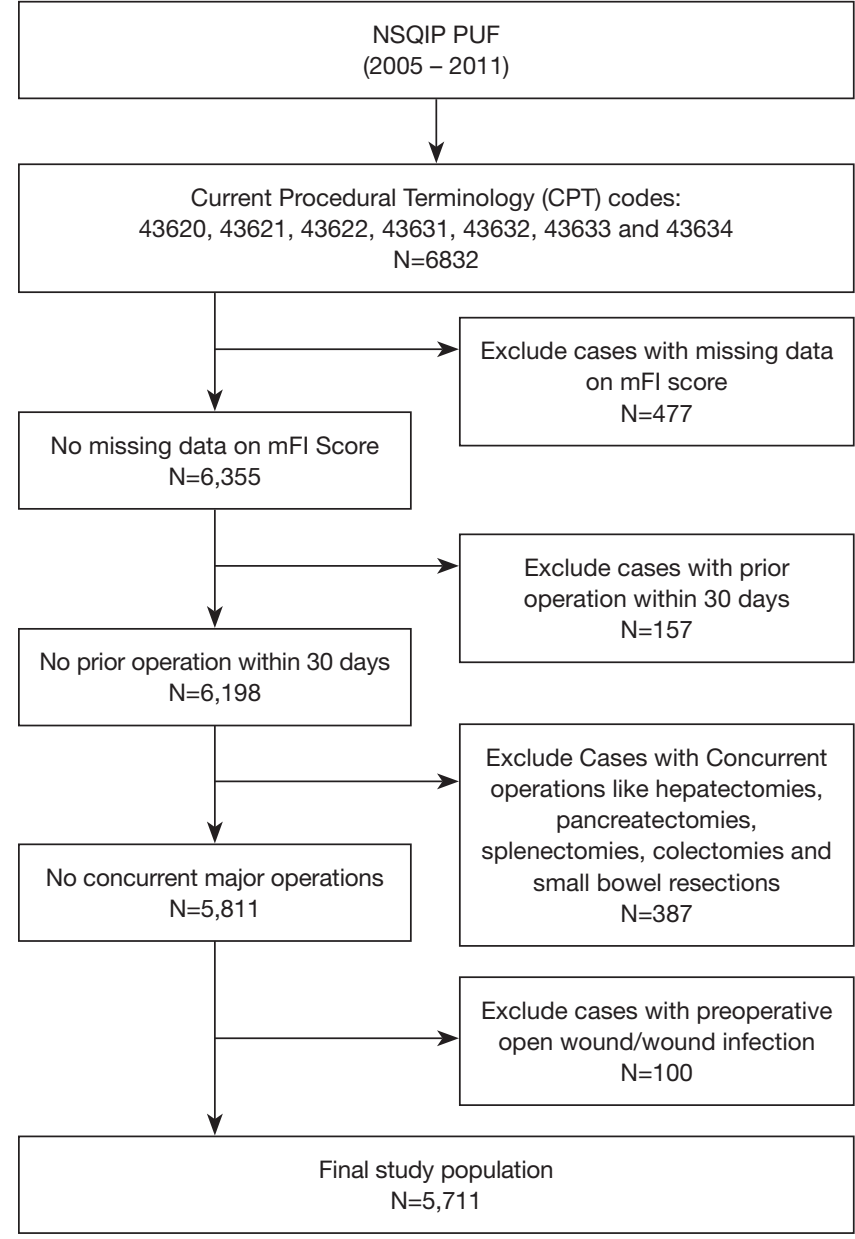

Figure 1 Flow diagram of patients selected for analysis. mFI, modified frailty index.

male patients, ASA scores $\geq 3$ and malignancy; and decreased percentages of body weight loss $(>10 \%$ in the last six months before the operation), preoperative chemotherapy, and preoperative radiotherapy. Further information of the patient's demographic and preoperative tests are summarized in Table 2. For the multivariable analysis we grouped the procedures in distal/partial gastrectomies, total gastrectomies and gastrectomy with pouch formation.

\section{Postoperative complications and estimates of covariate associations with mortality and serious morbidity}

Clavien IV complication occurred in 635 (11.1\%) patients during the first 30 postoperative days after the operation, and the 30-day mortality was 3.9\%. Table 3 and Figure 2 provide a breakdown of morbidity and mortality according to the frail groups. A significant increase in overall complications, Clavien IV complications, cardiopulmonary complications, sepsis related complications, failure to rescue from serious complications, and mortality were seen with an increased $\mathrm{mFI}$. However, no significant difference was found in the rate of organ space SSI between the mFI groups which indicates no surgical causes of postoperative complications and mortality. The prognostic value of the $\mathrm{mFI}$ score was confirmed also in the population with malignancy after performing a subgroup analysis (Table 3). Univariate regression analysis identified frailty as the strongest preoperative factor for having postoperative CLIVC (frail group: $\mathrm{OR}=5.1, \mathrm{CI}=3.94-6.59$ ) and mortality (frail group: $\mathrm{OR}=9.98, \mathrm{CI}=6.24-15.97$ ) (Table 4). On univariate analysis, CLIVC associated with age $\geq 65$, male gender, preoperative weight loss $>10 \%$, preoperative steroid use, hematocrit $<30$ and type of gastrectomy (total gastrectomy). Moreover, mortality was associated with age $\geq 65$, BMI $\geq 30$, preoperative weight loss $>10 \%$, preoperative chemotherapy, preoperative steroid use and hematocrit $<30$.

In multivariable regression analysis, after adjusting for confounders, low frail patients had more than $50 \%$ higher odds of having CLIVC and almost 100\% higher odds of 30-days mortality in comparison to non-frail. Intermediate frail patients had 140\% higher odds of CLIVC and 200\% higher odds of 30-day mortality. Frail patients had more than $280 \%$ higher odds of CLIVC and $410 \%$ higher odds of 30-day mortality. In multivariable logistic regression model, the preoperative factors which were consistently statistically significant in CLIVC are the frailty status, age $>65$, male gender, weight loss $>10 \%$, steroid use, hematocrit $<30$ and total gastrectomy. The corresponding factors for mortality are frailty, age $>65$, weight loss $>10 \%$, preoperative chemotherapy, preoperative steroid use and hematocrit $<30$.

Because $\mathrm{mFI}$ is not weighted and there was a possibility that every component of frailty calculation has different impact on postoperative morbidity and mortality, we analyzed the individual contribution of each frailty component toward the risk of CLIVC and mortality (Table 5). It appears that functional status, hypertension, CHF, MI, CS or angina or PCA history, COPD or pneumonia, PVD or Rest Pain and Impaired Sensorium are the mFI factors that steadily influence the final $\mathrm{mFI}$ score predictive value on both mortality and CLIVC. Diabetes Mellitus and TIA or CVA without neurologic deficit seems to affect the mFI score only on the CLIVC prediction. On the other hand CVA with neurologic deficits seems to affect the $\mathrm{mFI}$ score only 


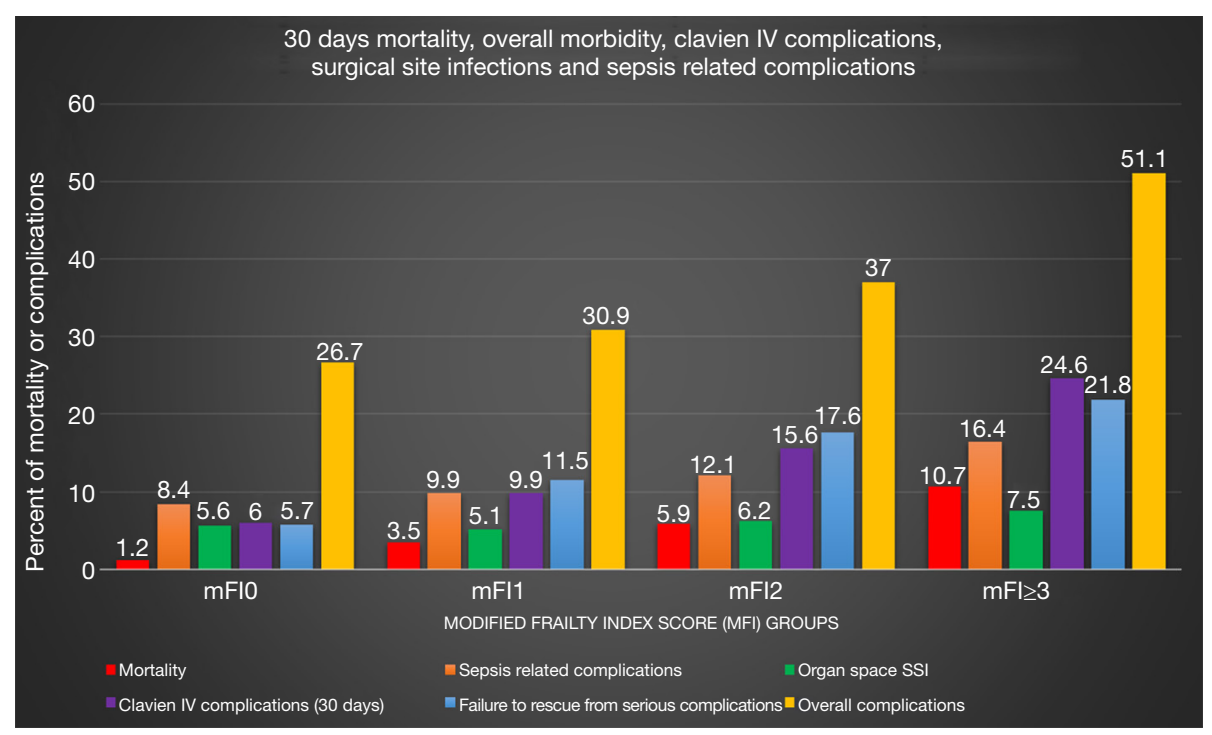

Figure 2 Observed 30 days mortality, overall morbidity, Clavien IV complications, surgical site infections and sepsis related complications by frailty group.

Table 4 Univariate and multivariate regression analysis of risk factors for postoperative Clavien IV complications and 30 days mortality

\begin{tabular}{|c|c|c|c|c|c|c|c|c|}
\hline \multirow{3}{*}{ Risk factors } & \multicolumn{4}{|c|}{ Univariate analysis } & \multicolumn{4}{|c|}{ Multivariate analysis } \\
\hline & \multicolumn{2}{|c|}{ Clavien IV } & \multicolumn{2}{|l|}{ Mortality } & \multicolumn{2}{|c|}{ Clavien IV } & \multicolumn{2}{|l|}{ Mortality } \\
\hline & uOR $(95 \% \mathrm{Cl})$ & $P$ value & uOR (95\% Cl) & $P$ value & aOR (95\% Cl) & $P$ value & aOR $(95 \% \mathrm{Cl})$ & $P$ value \\
\hline \multicolumn{9}{|l|}{ Frailty groups } \\
\hline No frailty & ref & & ref & & ref & & ref & \\
\hline Low frailty & $1.73(1.37-2.19)$ & $<0.001$ & 2.99 (1.89-4.77) & $<0.001$ & $1.51(1.18-1.94)$ & 0.001 & $1.94(1.18-3.19)$ & 0.009 \\
\hline Intermediate frailty & $2.89(2.26-3.69)$ & $<0.001$ & $5.18(3.24-8.28)$ & $<0.001$ & $2.4(1.84-3.13)$ & $<0.001$ & 3.01 (1.79-5.05) & $<0.001$ \\
\hline Frail & $5.1(3.94-6.59)$ & $<0.001$ & $9.98(6.24-15.97)$ & $<0.001$ & $3.88(2.91-5.16)$ & $<0.001$ & $5.12(3.03-8.65)$ & $<0.001$ \\
\hline Age $\geq 65$ & $2.05(1.72-2.43)$ & $<0.001$ & $4.615(3.26-6.53)$ & $<0.001$ & $1.43(1.19-1.75)$ & $<0.001$ & $3.08(2.09-4.53)$ & $<0.001$ \\
\hline Gender, male & $1.4(1.18-1.65)$ & $<0.001$ & $1.24(0.95-1.63)$ & 0.116 & $1.2(1.01-1.44)$ & 0.042 & - & - \\
\hline $\mathrm{BMI} \geq 30$ & $1.07(0.88-1.29)$ & 0.49 & $0.69(0.49-0.97)$ & 0.031 & - & & $0.87(0.61-1.25)$ & 0.451 \\
\hline Weight loss $>10 \%$ & $1.26(1.01-1.58)$ & 0.04 & $1.76(1.27-2.45)$ & 0.001 & $1.32(1.04-1.68)$ & 0.022 & $2.03(1.41-2.93)$ & $<0.001$ \\
\hline Chemotherapy & $0.77(0.49-1.21)$ & 0.26 & 1.99 (1.19-3.33) & 0.008 & - & & $2.38(1.38-4.1)$ & 0.002 \\
\hline Steroid use & $2.8(1.95-4.03)$ & $<0.001$ & 2.84 (1.66-4.84) & $<0.001$ & $2.52(1.72-3.71)$ & $<0.001$ & $2.30(1.28-4.13)$ & 0.005 \\
\hline $\mathrm{HCT}<30$ & $2.11(1.74-2.57)$ & $<0.001$ & $2.29(1.69-3.1)$ & $<0.001$ & 1.93 (1.57-2.37) & $<0.001$ & $1.92(1.37-2.68)$ & $<0.001$ \\
\hline \multicolumn{9}{|l|}{ Type of gastrectomy } \\
\hline Distal gastrectomy & ref & & ref & & ref & & - & - \\
\hline Total gastrectomy & $1.26(1.05-1.53)$ & 0.015 & $0.694(0.69-1.32)$ & 0.796 & $1.42(1.16-1.73)$ & $<0.001$ & - & - \\
\hline $\begin{array}{l}\text { Gastrectomy with } \\
\text { pouch formation }\end{array}$ & $1.21(0.79-184)$ & 0.39 & $0.84(0.39-1.82)$ & 0.664 & $1.37(0.87-2.15)$ & 0.171 & - & - \\
\hline
\end{tabular}

aOR, adjusted odds ratios; uOR, unadjusted odds ratios. 
Table 5 Unadjusted risk of Clavien IV complications and mortality for each individual frailty components

\begin{tabular}{|c|c|c|c|c|}
\hline \multirow{2}{*}{ Frailty components } & \multicolumn{2}{|c|}{ Mortality } & \multicolumn{2}{|c|}{ Clavien IV complications } \\
\hline & OR $(95 \% \mathrm{Cl})$ & $P$ value & OR (95\% Cl) & $P$ value \\
\hline FS & $7.19(5.36-9.66)$ & $<0.001$ & $4.51(3.63-5.59)$ & $<0.001$ \\
\hline Diabetes mellitus & $1.25(0.9-1.74)$ & 0.178 & $1.27(1.03-1.55)$ & 0.024 \\
\hline Hypertension & $2.49(1.85-3.37)$ & $<0.001$ & $1.64(1.38-1.94)$ & $<0.001$ \\
\hline $\mathrm{CHF}$ & $5.26(2.63-10.53)$ & $<0.001$ & $3.88(2.24-6.75)$ & $<0.001$ \\
\hline MI & $4.69(2.07-10.63)$ & $<0.001$ & $2.944(1.51-5.73)$ & 0.001 \\
\hline CS or Angina or PCA history & $2.39(1.73-3.32)$ & $<0.001$ & $2.09(1.68-2.59)$ & $<0.001$ \\
\hline COPD or pneumonia & $3.05(2.07-4.49)$ & $<0.001$ & $2.86(2.19-3.73)$ & $<0.001$ \\
\hline PVD or rest pain & $2.53(1.15-5.57)$ & 0.021 & $2.13(1.22-3.71)$ & 0.008 \\
\hline Impaired sensorium & $8.77(4.72-16.32)$ & $<0.001$ & $23.29(12.8-42.3)$ & $<0.001$ \\
\hline TIA or CVA without neurologic deficit & $1.56(0.89-2.73)$ & 0.117 & $2.0(1.44-2.78)$ & $<0.001$ \\
\hline CVA with neurologic deficits & $2.19(1.7-4.12)$ & 0.015 & $1.27(0.78-2.08)$ & 0.334 \\
\hline
\end{tabular}

FS: functional status-dependent vs. non-dependent: this variable focuses on the patient's abilities to perform activities of daily living (ADLs) in the 30 days prior to surgery. Activities of daily living are defined as 'the activities usually performed in the course of a normal day in a person's life'. ADLs include: bathing, feeding, dressing, toileting, and mobility. CHF, congestive heart failure; MI, myocardial infarction history; CS, history of cardiac surgery; PCA, percutaneous coronary angioplasty; PVD, peripheral vein disease; TIA, transient ischemic attack; CVA, cerebrovascular accident.

on the mortality prediction and not on CLIVC prediction.

Finally, the mFI score demonstrates good discrimination for both 30-day mortality (AUROC =0.703, 95\% CI: 0.669 0.738 ) and Clavien IV complications (AUROC $=0.65,95 \%$ CI: 0.627-0.673). Furthermore, the discriminative ability of the model with adjustment of covariates it seems to be even better for both 30-day mortality (AUROC $=0.773,95 \%$ CI: 0.742-0.805) and Clavien IV complications (AUROC $=0.692,95 \%$ CI: $0.67-0.714)$.

\section{Discussion}

Gastrectomy remains as the main curative treatment option for any patient with either malignant or benign gastric tumor. However, gastrectomy is associated with significant morbidity and mortality $(2,3)$. These can be attributed to: the complexity of the particular operation and also to the fact that patient undergoing gastrectomy are usually older with many comorbidities $(1,11)$. Models used in the past lacking predictive value or are too laborious to use (11). Moreover, the majority of recent studies for preoperative risk estimation in gastrectomy have focused on either single risk factors or single postoperative outcomes, both of which have limited clinical use $(2,12,13)$. We used a simplified method of calculation mFI tailored to the ACS-NSQIP (5).

Our results provide compelling evidence that $\mathrm{mFI}$ is a powerful predicting model for 30-day mortality, CLVIC and postoperative failure to rescue after a serious complication. The present study revealed that frail patients undergoing gastrectomy have almost 290\% higher odds of having a CLIVC complication and more that $400 \%$ higher odds of having postoperative mortality. Also, we found that frail patients undergoing gastrectomy have significantly higher rates of any complication, cardiopulmonary complications, sepsis-related complications and failure to rescue from a serious complication. However, frail patients undergoing gastrectomy were not found to have significantly higher rates of postoperative organ space SSI which would suggest anastomotic leaks. This supports the idea of reduced physiologic reserves, and not technical errors as the root cause. Our results are analogous to existing studies of $\mathrm{mFI}$ for other operations like hepatectomies, pancreatectomies, necrosectomies and gynecologic operations. The $\mathrm{mFI}$ score is a highly useful tool for daily clinical use (14-17).

This study has several limitations, both in data extraction and database design. First, the preoperative variables are predefined for specific comorbidities and laboratories, and much critical information is lacking. For example, the 
information regarded the total glycated hemoglobin for diabetic patients is not available. Therefore, we cannot differentiate patients with poorly controlled diabetes mellitus from those with optimal control. Also, the postoperative outcomes are limited to the postoperative 30day period. Furthermore, NSQIP database has significant limitations on technical-related complications report, and these complications can incorrectly be attributed to preoperative patient's frail status (18). Finally, our study is subjected to all potential limitations of a retrospective study.

Future work can be focused on incorporating more factors in the model and adding details such as genetic information, laboratory values as well as social such as smoking, alcohol or illicit drug use of the patient. Furthermore, the mFI score can be applied to new mobile or computer applications, and play a fundamental role in the decision-making process, something already proposed by Koble et al. (17).

In conclusion, $\mathrm{mFI}$ incorporation in preoperative assessment of patient undergoing gastrectomy adds valuable information regarding the postoperative morbidity, mortality and failure to rescue after a serious complication.

\section{Acknowledgments}

The American College of Surgeons National Surgical Quality Improvement Program and the hospitals participating in the ACS NSQIP are the source of the data used herein; they have not verified and are not responsible for the statistical validity of the data analysis or the conclusions derived by the authors. We would like to thank Dr. Andres for assistance with statistical analysis.

Funding: None.

\section{Footnote}

Provenance and Peer Review: This article was a free submission to the journal. The article has undergone external peer review.

Conflicts of Interest: All authors have completed the ICMJE uniform disclosure form (available at http://dx.doi. org/10.21037/tgh.2020.01.07). The authors have no conflicts of interest to declare.

Ethical Statement: The authors are accountable for all aspects of the work in ensuring that questions related to the accuracy or integrity of any part of the work are appropriately investigated and resolved. The NSQIP data base is a de-identified patient database and consequently our study was exempt from institutional review board (IRB) approval.

Open Access Statement: This is an Open Access article distributed in accordance with the Creative Commons Attribution-NonCommercial-NoDerivs 4.0 International License (CC BY-NC-ND 4.0), which permits the noncommercial replication and distribution of the article with the strict proviso that no changes or edits are made and the original work is properly cited (including links to both the formal publication through the relevant DOI and the license). See: https://creativecommons.org/licenses/by-nc-nd/4.0/.

\section{References}

1. Birkmeyer JD, Sun Y, Wong SL, et al. Hospital volume and late survival after cancer surgery. Ann Surg 2007;245:777-83.

2. Martin AN, Das D, Turrentine FE, et al. Morbidity and Mortality After Gastrectomy: Identification of Modifiable Risk Factors. J Gastrointest Surg 2016;20:1554-64.

3. Papenfuss WA, Kukar M, Oxenberg J, et al. Morbidity and mortality associated with gastrectomy for gastric cancer. Ann Surg Oncol 2014;21:3008-14.

4. Le Manach Y, Collins G, Rodseth R, et al. Preoperative Score to Predict Postoperative Mortality (POSPOM): Derivation and Validation. Anesthesiology 2016;124:570-9.

5. Velanovich V, Antoine H, Swartz A, et al. Accumulating deficits model of frailty and postoperative mortality and morbidity: its application to a national database. J Surg Res 2013;183:104-10.

6. Program ACoSNSQI. ACS NSQIP 2017 Available online: https://www.facs.org/quality-programs/acs-nsqip

7. Augustin T, Burstein MD, Schneider EB, et al. Frailty predicts risk of life-threatening complications and mortality after pancreatic resections. Surgery 2016;160:987-96.

8. Dindo D, Demartines N, Clavien PA. Classification of surgical complications: a new proposal with evaluation in a cohort of 6336 patients and results of a survey. Ann Surg 2004;240:205-13.

9. Ghaferi AA, Birkmeyer JD, Dimick JB. Variation in hospital mortality associated with inpatient surgery. $\mathrm{N}$ Engl J Med 2009;361:1368-75.

10. Silber JH, Williams SV, Krakauer H, et al. Hospital and 
patient characteristics associated with death after surgery. A study of adverse occurrence and failure to rescue. Med Care 1992;30:615-29.

11. Ueno D, Matsumoto H, Kubota H, et al. Prognostic factors for gastrectomy in elderly patients with gastric cancer. World J Surg Oncol 2017;15:59.

12. Kim SH, Son SY, Park YS, et al. Risk Factors for Anastomotic Leakage: A Retrospective Cohort Study in a Single Gastric Surgical Unit. J Gastric Cancer 2015;15:167-75.

13. Choe YR, Joh JY, Kim YP. Association between frailty and readmission within one year after gastrectomy in older patients with gastric cancer. J Geriatr Oncol 2017;8:185-9.

14. Louwers L, Schnickel G, Rubinfeld I. Use of a simplified frailty index to predict Clavien 4 complications and mortality after hepatectomy: analysis of the National Surgical Quality Improvement Project database. Am J

doi: $10.21037 / \operatorname{tgh} .2020 .01 .07$

Cite this article as: Zorbas KA, Velanovich V, Esnaola NF, Karachristos A. Modified frailty index predicts complications and death after non-bariatric gastrectomies. Transl Gastroenterol Hepatol 2021;6:10.
Surg 2016;211:1071-6.

15. Mogal H, Vermilion SA, Dodson R, et al. Modified Frailty Index Predicts Morbidity and Mortality After Pancreaticoduodenectomy. Ann Surg Oncol 2017;24:1714-21.

16. George EM, Burke WM, Hou JY, et al. Measurement and validation of frailty as a predictor of outcomes in women undergoing major gynaecological surgery. BJOG 2016;123:455-61.

17. Kolbe N, Bakey S, Louwers L, et al. Predictors of Clavien 4 Complications and Mortality After Necrosectomy: Analysis of the NSQIP Database. J Gastrointest Surg 2015;19:1086-92.

18. Shah R, Velanovich V, Syed Z, et al. Limitations of patientassociated co-morbidity model in predicting postoperative morbidity and mortality in pancreatic operations. J Gastrointest Surg 2012;16:986-92. 